\title{
Heparin-Binding Copolymer as a Complete Antidote for Low-Molecular-Weight Heparins in Rats
}

\author{
Bartlomiej Kalaska, (DJoanna Miklosz, (D) Kamil Kamiński, DJustyna Swieton, \\ (D)Aleksandra Jakimczuk, (D) Shin-Ichi Yusa, DDariusz Pawlak, (D) Maria Nowakowska, \\ (D) Krzysztof Szczubiałka, and (1)Andrzej Mogielnicki \\ Department of Pharmacodynamics, Medical University of Bialystok, Bialystok, Poland (B.K., J.M., J.S., A.J., D.P., A.M.); Faculty \\ of Chemistry, Jagiellonian University, Krakow, Poland (K.K., M.N., K.S.); and Department of Applied Chemistry, Graduate School \\ of Engineering, University of Hyogo, Himeji, Hyogo, Japan (S.-I.Y.)
}

Received September 30, 2019; accepted January 9, 2020

\begin{abstract}
Bleeding resulting from the application of low-molecular-weight heparins (LMWHs) may be treated with protamine sulfate, but this treatment lacks efficiency; its action against antifactor Xa activity is limited to $\sim 60 \%$. Moreover, protamine sulfate can cause life-threatening hypersensitivity reactions. We developed diblock heparin-binding copolymer ( $\mathrm{HBC}$ ), which can neutralize the anticoagulant activity of parenteral anticoagulants. In the present study, we explored the safety profile of HBC and its potential to reverse enoxaparin, nadroparin, dalteparin, and tinzaparin in human plasma and at in vivo conditions. HBCLMWH complexes were characterized using zeta potential, isothermal titration calorimetry, and dynamic light scattering. The rat cardiomyocytes and human endothelial cells were used for the assessment of in vitro toxicity. Male Wistar rats were observed for up to 4 days after HBC administration for clinical evaluation, gross necropsy, and biochemistry and histopathological analysis. Rats were treated with LMWHs alone or followed by short-time intravenous infusion of HBC, and bleeding time and antifactor Xa activity were measured. HBC completely reversed antifactor $\mathrm{Xa}$ activity prolonged
\end{abstract}

\section{Introduction}

Low-molecular-weight heparins (LMWHs) represent a group of parenteral anticoagulants for prevention and treatment of several thrombotic disorders, that is, unstable angina, myocardial infarction, and deep vein or cancer-associated thrombosis. The global parenteral anticoagulant market is dominated by LMWHs and is still growing. LMWHs offer numerous advantages over standard unfractionated heparin (UFH), including more predictable pharmacodynamics and pharmacokinetics, less restrictive monitoring, safer subcutaneous administration, and more favorable overall safety

This work was funded by National Science Centre, Poland Grant 2016/21/B/ ST5/00837. The efficacy studies in human plasma were supported by Medical University of Bialystok project no. SUB/2/DN/19/003/2211.

https://doi.org/10.1124/jpet.119.262931. in vitro by all LMWHs with an optimal weight ratio of 2.5:1. The complexes of HBC-LMWHs were below $5 \mu \mathrm{m}$. We observed no effects on the viability of cardiovascular cells treated with HBC at concentrations up to $0.05 \mathrm{mg} / \mathrm{ml}$. Single doses up to $20 \mathrm{mg} / \mathrm{kg}$ of HBC were well tolerated by rats. HBC completely reversed the effects of LMWHs on bleeding time and antifactor $\mathrm{Xa}$ activity in vivo after 20 minutes and retained $\sim 80 \%$ and $\sim 60 \%$ of reversal activity after 1 and 2 hours, respectively. Well-documented efficacy and safety of HBC both in vitro and in vivo make this polymer a promising candidate for LMWHs reversal.

\section{SIGNIFICANCE STATEMENT}

Over the last decade, there has been significant progress in developing antidotes for the reversal of anticoagulants. Until now, there has been no effective and safe treatment for patients with severe bleeding under low-molecular-weight heparin therapy. Based on our in vitro and in vivo studies, heparin-binding copolymer seems to be a promising candidate for neutralizing all clinically relevant low-molecular-weight heparins.

ABBREVIATIONS: CrV, crystal violet; DLS, dynamic light scattering; HBC, heparin-binding copolymer; HUVEC, human umbilical vein endothelial cell; ITC, isothermal titration calorimetry; LMWH, low-molecular-weight heparin; PEG, poly(ethylene glycol); PMAPTAC, poly(3(methacryloylamino)propyl trimethylammonium chloride); UFH, unfractionated heparin; UHRA, Universal Heparin Reversal Agent. 
frequency of bleeding episodes (van Rein et al., 2017). In the most clinical trials involving the management of thrombotic disorders with LMWHs, the incidence of major bleeding was between $1.5 \%$ and $4.7 \%$ and depended mainly on the type and duration of anticoagulation used and on the indication claimed (Simonneau et al., 1997; Hull et al., 2000; Turpie et al., 2002; Petersen et al., 2004; Mismetti et al., 2005; Crowther and Warkentin, 2008).

Severe bleeding related to LMWHs may be treated with intravenous administration of protamine sulfate. Protamine sulfate fully neutralizes LMWHs' antifactor IIa activity, but its action against antifactor $\mathrm{Xa}$ activity is limited to $\sim 60 \%$ (Garcia et al., 2012). In the retrospective study, LMWH-induced active bleeding was effectively stopped in $66 \%$ of patients who received protamine (van Veen et al., 2011). Protamine sulfate can also cause life-threatening hypersensitivity reactions, such as hypotension, pulmonary hypertension, noncardiogenic pulmonary edema, cardiovascular collapse, and pulmonary vasoconstriction. Additionally, several risk factors for severe hypersensitivity reactions to protamine have been described, including rapid administration, previous administration of protamine or protamine insulin, fish allergy, left ventricular dysfunction, and previous vasectomy (Sokolowska et al., 2016). Therefore, each administration of protamine should be carefully considered, and better alternatives for LMWHs reversal are required.

Several new reversal agents for LMWHs (mainly enoxaparin and tinzaparin) are being developed. Ciraparantag is a cationic, synthetic small molecule designed as an antidote for direct oral anticoagulants, UFH, and enoxaparin (Laulicht et al., 2013). Andexanet alfa, a recombinant derivative of factor $\mathrm{Xa}$, has been designed to reverse the anticoagulant effects of direct and indirect factor Xa inhibitors ( $\mathrm{Lu}$ et al., 2013). However, andexanet alfa has received approval only for uncontrolled and life-threatening bleeding in patients treated with oral anticoagulants: apixaban and rivaroxaban (Heo, 2018). Another antidote currently in the preclinical phase of development is Universal Heparin Reversal Agent (UHRA). UHRA, a synthetic dendrimeric polymer, has been designed to reverse the anticoagulant activity of all available heparins (Shenoi et al., 2014; Kalathottukaren et al., 2017a). In a recently published in vitro comparative study, UHRA directly bound both UFH and enoxaparin. Andexanet alfa and ciraparantag bound the above heparins relatively weakly. For andexanet alfa and UHRA, binding correlated positively with the neutralization activity. The authors did not observe the reversal activity of ciraparantag in assays typically used to assess anticoagulant effects (Kalathottukaren et al., 2018). None of the three above antidotes have been approved by the Food and Drug Administration for the management of LMWH-induced bleeding.

Therefore, to address the significant and still-unmet clinical need, we developed a heparin-binding polymer (HBC) [poly(ethylene glycol) (PEG) 41-poly(3-(methacryloylamino)propyl trimethylammonium chloride) (PMAPTAC) 53], a reversal agent for parenteral anticoagulants. HBC is a diblock synthetic polymer that directly binds anticoagulant molecules and effectively neutralizes their effects in the living organism (Kalaska et al., 2016, 2018, 2019). In this work, we further explore the potential of HBC to safely reverse LMWHs widely used in the clinical practice (i.e., enoxaparin, nadroparin, dalteparin, and tinzaparin) at in vivo conditions and in human plasma.

\section{Materials and Methods}

In Vitro Neutralization Assay. Enoxaparin, nadroparin, dalteparin, and tinzaparin were purchased from Sanofi-Aventis (France), Aspen Notre Dame de Bondueville (France), Pfizer Europe MA EEIG (UK), and Sigma-Aldrich (Germany), respectively. The neutralization of the above LMWHs was analyzed by measuring antifactor Xa activity in a 96-well plate reader (Synergy HTX, BioTek) according to the modified kit manufacturer instructions (BioMedica Diagnostics, Canada), as described previously (Kalaska et al., 2016). In brief, sodium citrate-anticoagulated pooled plasma was obtained from healthy rats or human volunteers by centrifugation of whole blood at $3500 \mathrm{~g}$ for 20 minutes at $4^{\circ} \mathrm{C}$. Two hundred microliters of pooled plasma were mixed with $10 \mu \mathrm{l}$ of LMWHs $(20 \mu \mathrm{g} / \mathrm{ml}$ of plasma for enoxaparin and tinzaparin; $2 \mathrm{U} / \mathrm{ml}$ for nadroparin and dalteparin). After 150 seconds of incubation $\left(37^{\circ} \mathrm{C}\right), 10 \mu \mathrm{l}$ of a solution containing increasing concentrations of $\mathrm{HBC}(0.01-0.1 \mathrm{mg} / \mathrm{ml}$ of plasma) was added. After another 150 seconds of incubation $\left(37^{\circ} \mathrm{C}\right)$, antifactor $\mathrm{Xa}$ activity was measured at $405 \mathrm{~nm}$. All the procedures involving healthy human volunteers were approved by the Local Ethics Committee of the Medical University of Bialystok (Permit No. R-I-002/193/2019).

Zeta Potential Measurements. Zeta potential of all HBCLMWH complexes in ratios based on the in vitro neutralization assay was measured using a Zetasizer Nano-ZS (Malvern Instruments, UK). The samples were prepared in PBS ( $\mathrm{pH} 7.4$ ), filtered through a Chromafil filter $(0.45 \mu \mathrm{m})$, and measured at $25^{\circ} \mathrm{C}$. The data were analyzed using the Malvern software.

Isothermal Titration Calorimetry Measurements. All isothermal titration calorimetry (ITC) measurements were made using Malvern MICRO-CAL PEAQ-ITC. HBC was dissolved in PBS; its concentration was $12 \mathrm{~g} / \mathrm{l}(0.001078 \mathrm{~mol} / \mathrm{l})$. LMWHs' commercial solutions were also diluted in PBS to concentrations of about 125 $\mathrm{U} / \mathrm{ml}$ or $1.25 \mathrm{mg} / \mathrm{ml}$. The measurement temperature was $25^{\circ} \mathrm{C}$, and the mixing speed was $750 \mathrm{rpm}$. The measuring chamber contained $270 \mu \mathrm{l}$ of LMWH solution to which 19 portions of HBC solution, $2 \mu \mathrm{l}$ each, were added.

The binding constant $\left(K_{a}\right)$ between a molecule of HBC and a free binding site of a respective LMWH was calculated using the formula:

$$
K_{a}=\frac{[\text { bound } H B C]}{[\text { free binding sites of } L M W H][\text { free } H B C]}
$$

Dynamic Light Scattering Measurements. The size of complexes formed by HBCs and LMWHs in ratios based on the in vitro neutralization was studied with the dynamic light scattering (DLS) technique. LMWHs were dissolved in PBS and bovine serum. To that mixture, the HBC solutions in PBS were added, and the formation of complexes was monitored by DLS using a Zetasizer Nano-ZS (Malvern Instruments).

Cell Lines Used and Cell Viability Assay. Primary human umbilical vein endothelial cells (HUVECs) (American Type Culture Collection-1730) and nontransformed embryonic rat heart-derived cells [H9c2(2-1), American Type Culture Collection CRL-1446] were used as healthy control cell lines for assessment of the cardiovascular system toxicity of HBC. HUVECs were cultured in Medium 199 containing $20 \%$ fetal bovine serum with cell growth supplements at $37^{\circ} \mathrm{C}$ in the atmosphere containing $5 \% \mathrm{CO}_{2}$ and at $95 \%$ humidity. All experiments were performed with mycoplasma-free cells. The ATPlite luminescence test (Perkin Elmer Life Sciences) was used to assess the proliferation and viability of HUVECs in vitro. Cells were seeded in 96-well plates, and after overnight incubation, the cells were treated with increasing concentrations of HBC for 72 hours. Cells treated with formulation buffer were used as a control. H9c(2-1) cells were cultured in Dulbecco's modified Eagle's medium containing $10 \%$ fetal bovine serum at $37^{\circ} \mathrm{C}$ in the atmosphere containing $5 \% \quad \mathrm{CO}_{2}$ and at $90 \%$ humidity. The experiment was carried out for 24 hours after adding HBC to cell media. To assess cell viability, crystal violet $(\mathrm{CrV})$ assay was used (Popiołek et al., 2019). Briefly, cells were seeded into 
24-well plates at the density of about $1.1 \times 10^{4}$ cells $/ \mathrm{cm}^{2}$. After incubation, the medium was removed, and the cells were washed and fixed using $1 \mathrm{ml}$ of $4 \% \mathrm{v} / \mathrm{v}$ formaldehyde/PBS and treated for 2 minutes with a $\mathrm{CrV}$ solution. Then, unbound $\mathrm{CrV}$ was removed by rinsing with water. After drying, the destaining solution was added to each well and left for 30 minutes. Finally, absorbance of the obtained solution at $540 \mathrm{~nm}$ was measured, which was proportional to the number of living cells.

Animals. Male Wistar rats were obtained from the Centre of Experimental Medicine in the Medical University of Bialystok, grouped into cages as appropriate, and allowed to have ad libitum access to sterilized tap water and standard chow in specific pathogenfree conditions. All the procedures involving animals were approved by the Local Ethical Committee on Animal Testing (Permit Nos. for the safety study: $38 / 2017$ and 85/2019; Permit Nos. for the efficacy study: 15/2013 and 60/2018) and conducted in accordance to the Directive 2010/63/EU of the European Parliament and the Council on the protection of animals, Animal Research: Reporting of In Vivo Experiments guidelines, and national laws.

Safety Study in Rats. Thirty-five male Wistar rats were randomly assigned to five groups. HBC was administered into the tail vein of six rats at the initial dose of $5 \mathrm{mg} / \mathrm{kg}$. The rats were observed continuously for the first 30 minutes after $\mathrm{HBC}$ administration for clinical signs of toxicity. Then they were kept under observation for up to 4 days after $\mathrm{HBC}$ administration (intermittently for 4 hours and then at 6, 24, 48, 72, and 96 hours after HBC administration). Body weights were recorded before HBC administration and before the gross necropsy on day 4 . Following a minimum of 1 day without severe clinical findings, HBC was administered to a group of six rats at a dose of $10 \mathrm{mg} / \mathrm{kg}$. These rats were observed for clinical signs of toxicity. This procedure was repeated in six rats treated with $\mathrm{HBC}$ at a dose of $20 \mathrm{mg} / \mathrm{kg}$ and $10 \mathrm{rats}$ treated with $\mathrm{HBC}$ at a dose of $40 \mathrm{mg} / \mathrm{kg}$. PBS was also administered to seven animals as a control group. On the 4th day, four rats treated with PBS and four rats treated with $\mathrm{HBC}$ at the dose of $40 \mathrm{mg} / \mathrm{kg}$ were anesthetized by an intraperitoneal injection of pentobarbital (45 mg/kg), blood was collected from the heart and centrifuged, and serum was used for the measurement of alanine aminotransferase and aspartate aminotransferase activities and total bilirubin, urea, and creatinine concentrations. Immediately after the blood collection, the whole liver, lungs, kidney, and thymus were removed for routine histological analysis using hematoxylin and eosin staining.

Efficacy Study in Rats. In the first procedure, 56 male Wistar rats were randomly assigned to seven groups, anesthetized by an intraperitoneal injection of pentobarbital $(45 \mathrm{mg} / \mathrm{kg})$, and placed in a supine position on a heated operating table. The body weights ranged from 200 to 250 g just before anesthesia induction. Nadroparin (800 U/kg), dalteparin $(800 \mathrm{U} / \mathrm{kg})$, or tinzaparin $(8 \mathrm{mg} / \mathrm{kg})$ was administered into the right femoral vein 10 minutes before the measurement of bleeding time. HBC $(20 \mathrm{mg} / \mathrm{kg})$ was intravenously infused (5 minutes) after LMWH administration. Bleeding time was measured as described previously (Kalaska et al., 2015). If bleeding was still present at the end of the 480 -second observation period, a value of 480 seconds was ascribed for the sake of statistical analysis. Twenty minutes after anticoagulant administration, antifactor Xa activity was analyzed with ELISA technique using a microplate reader (Synergy HTX, BioTek) according to the kit manufacturer instructions (BioMedica Diagnostics). If antifactor Xa activity was more than or equal to $1 \mathrm{U} / \mathrm{mg}$, a value of $1 \mathrm{U} / \mathrm{mg}$ was ascribed for the sake of statistical analysis.

In the second procedure, 43 male Wistar rats were randomly divided into six groups and anesthetized in the same manner as described in the first procedure. Rats were weighted just before anesthesia induction and the body weights ranged from 190 to 220 g. Nadroparin and HBC were also administered in the same doses and manner as described in the first procedure. Bleeding time was measured 10 minutes before blood collection. Antifactor Xa activity was analyzed 1 or 2 hours after anticoagulant administration. Bleeding time and antifactor Xa activity were measured as described above.
Statistical Analysis. In the in vivo study, $n$ refers to the number of animals in each experimental group. For each test, the experimental unit was an individual animal. The data are shown as mean \pm S.D. or median with lower and upper limits and analyzed with GraphPad Prism 6 software (GraphPad Prism 6 software) using the unpaired Student's $t$ test or Mann-Whitney test. $P$ values less than 0.05 were considered significant.

\section{Results}

The Synthesis of HBC (PEG41-PMAPTAC53) by Controlled Radical Polymerization. The synthesis of HBC by reversible-addition fragmentation chain transfer polymerization technique and selection strategy of the leading compound was previously described (Kalaska et al., 2016). Reversible-addition fragmentation chain transfer is one of the controlled/living radical polymerization processes, allowing synthesis of very well-defined polymers in terms of molecular weight and molecular weight dispersity. Briefly, a diblock copolymer with the degrees of polymerization of PEG and PMAPTAC blocks equal to 41 and 53, respectively, was synthesized (Fig. 1). The cationic PMAPTAC198 homopolymer was used as a control to assess the toxicity-reducing activity of the PEG block.

Cell Viability Reduction. HUVECs and cardiomyocytes were treated with the diblock HBC and a cationic PMAPTAC198 homopolymer. HUVEC viability was not negatively affected by HBC and PMAPTAC198 at concentrations up to $0.05 \mathrm{mg} / \mathrm{ml}$. Both studied polymers significantly impaired HUVEC viability at concentrations of $0.15 \mathrm{mg} / \mathrm{ml}$ (Fig. 2A). The conjugation of PMAPTAC with PEG significantly reduced the negative effect of the polycation PMAPTAC block on cardiomyocyte viability (Fig. 2B). At $0.15 \mathrm{mg} / \mathrm{ml}$, the maximum concentration studied, the viability of the cardiomyocytes treated with $\mathrm{HBC}$ was still about $100 \%$, whereas the viability of those treated with PMAPTAC198 decreased to about 50\%.

Efficacy in the In Vitro Study. HBC concentrationdependently and completely reversed antifactor Xa activity in vitro of enoxaparin, nadroparin, dalteparin, and tinzaparin in rat and human sodium citrate-anticoagulated plasma with an optimal ratio of $0.05 \mathrm{mg}$ of the polymer for $0.02 \mathrm{mg}$ of both enoxaparin and tinzaparin and $2 \mathrm{U}$ of both dalteparin and nadroparin (Fig. 3). We chose the concentration and dose of HBC for further in vitro and in vivo studies based on the estimated ratios: $2.5 \mathrm{mg}$ of $\mathrm{HBC}$ per $1 \mathrm{mg}$ of enoxaparin and tinzaparin and $2.5 \mathrm{mg}$ of HBC per $100 \mathrm{U}$ of dalteparin and nadroparin.

Zeta Potential of HBC-LMWH Complexes. Based on the effective neutralization ratios found, we next investigated the zeta potential of the HBC-LMWH complexes. In all cases, the zeta potential of complexes had a value close to zero (Table 1). The polycation completely neutralized the negative charge of LMWH molecules. Such a result was to be expected when the proportion of polymers in the mixture corresponded to the total binding of LMWH by polycation. DLS measurement showed a relatively large diameter of complexes due to potential values close to zero and the lack of electrostatic stabilization of the particles. In the case of tinzaparin, the complexes were the smallest.

Calorimetric Measurements and Thermodynamic Parameters of the Complexation Reaction. Thermodynamic parameters of the interactions between all studied LMWHs and HBC are given in Table 1, and the integral 
<smiles>COCC[14C](C)(C)OC(=O)CCC(C)(C)CC(C)(CC(C)(C)SC(=S)c1ccccc1)C(=O)NCCC[N+](C)(C)C</smiles>

Fig. 1. Chemical structure of HBC (PEG41-PMAPTAC53) (Kalaska et al., 2016). enthalpies of interaction are shown in Fig. 4. For enoxaparin and nadroparin, the drop inflection points in the respective curves are less clearly defined than for dalteparin and tinzaparin (Fig. 4, A and B vs. Fig. 4, C and D), which may be due to higher dispersity of the molecular weight of the former LMWHs.

The Size of LMWH-HBC Complexes. The size of complexes formed by LMWHs and HBCs in PBS and bovine serum was studied using DLS technique. The particle size of complexes in PBS and serum have diameters increasing in the following order: tinzaparin (120 and $220 \mathrm{~nm})$, dalteparin (0.5 and $1 \mu \mathrm{m}$ ), enoxaparin ( 0.8 and $5 \mu \mathrm{m}$ ), and nadroparin (in both cases, two populations $0.4 />5$ and $0.2 />5 \mu \mathrm{m}$ ). The presence of the polycation itself in the serum only slightly affected the size of the objects present in the mixture, and this effect can be considered negligible. The particle size of the complex formed in the serum was, in all cases, smaller than the diameter of the capillary blood vessels. A relatively large dimension equal to a fraction of the size of blood cells will promote the rapid elimination of complexes from the blood system (Fig. 5).

Safety in Animals. There were no significant HBCrelated effects on the body weight gain in rats treated for up to 4 days at the doses of 5,10 , and $20 \mathrm{mg} / \mathrm{kg}$. A $5 \%$ reduction in body weight occurred in the group treated with $\mathrm{HBC}$ at the dose of $40 \mathrm{mg} / \mathrm{kg}$. Administration of $\mathrm{HBC}$ at the dose of $20 \mathrm{mg} / \mathrm{kg}$ resulted in slightly decreased locomotive activity and apathy at approximately 3 hours postdose in $50 \%$ of rats.
We observed transient mild nasal swelling and edema of the limbs in five rats. All rats treated with $\mathrm{HBC}$ at the dose of $40 \mathrm{mg} / \mathrm{kg}$ had decreased locomotive activity and apathy (both 10-15 minutes following HBC administration); $40 \%$ of rats had an increased thirst (14-20 minutes following HBC administration); $50 \%$ of rats had an increased secretion of porphyrins ( 2 to 3 hours following $\mathrm{HBC}$ administration). All these findings were resolved within approximately 24 hours postdose. Furthermore, mild nasal swelling and edema of the limbs were observed in $40 \%$ of rats treated with $\mathrm{HBC}$ at the dose of $40 \mathrm{mg} / \mathrm{kg}$. These findings appeared immediately postdose and were resolved within approximately 6 hours. $\mathrm{HBC}$ at the dose of $40 \mathrm{mg} / \mathrm{kg}$ resulted in kidney congestion and color heterogeneity in $40 \%$ of rats. Gross necropsy findings in $30 \%$ of rats included the presence of urinary white sediment. Severe gross necropsy findings occurred in one rat and included lung congestion, kidney congestion, color heterogeneity, thymus congestion, and liver abnormalities. All rats survived the experiment (Table 2). We found higher serum concentrations of urea and creatinine in two out of four rats treated with HBC at the dose of $40 \mathrm{mg} / \mathrm{kg}$ (Table 3). The same two rats presented significant histopathological changes of acute tubular necrosis in kidney tissue, whereas two others presented mild tubular toxicity, which should not impair renal function. There were small areas of slight congestion and inflammatory cell infiltration in the lungs. HBC did not cause significant histopathological changes in other studied tissues (Fig. 6).
A

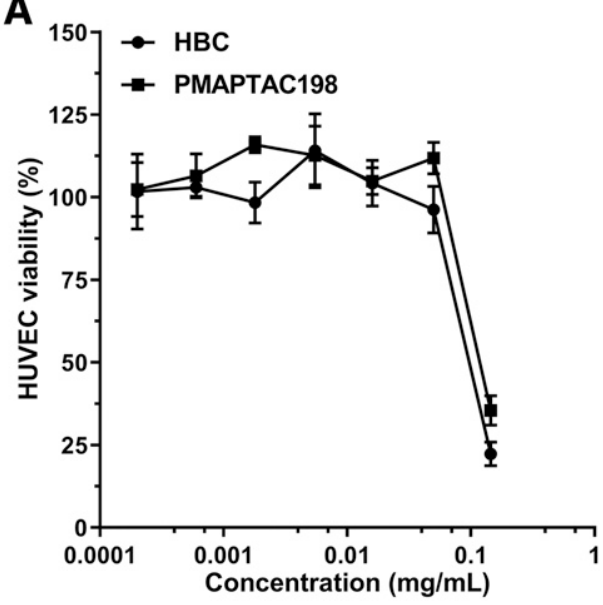

B

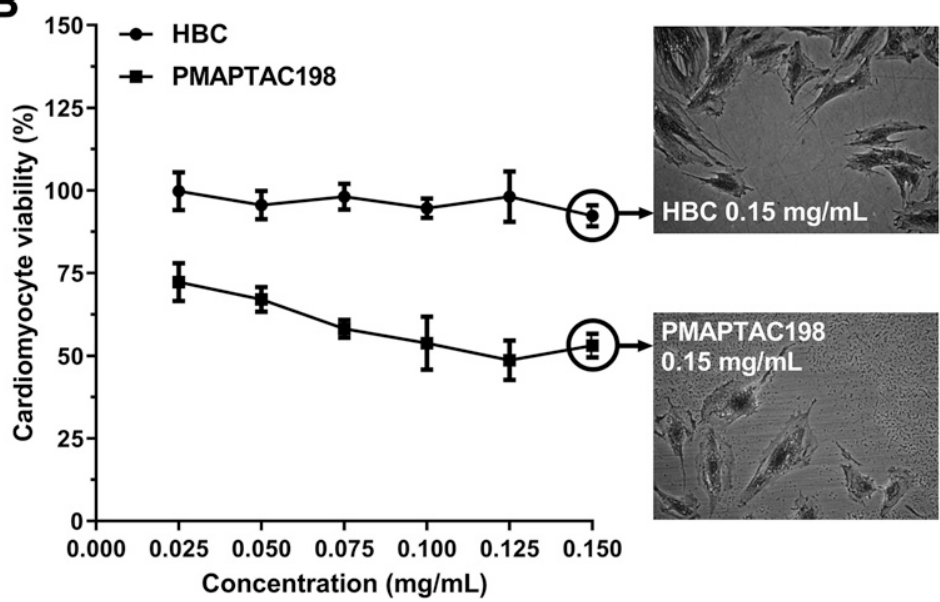

Fig. 2. Effect of HBC and PMAPTAC198 on HUVECs (A) and cardiomyocyte viability (B). Results are shown as mean \pm S.D. 

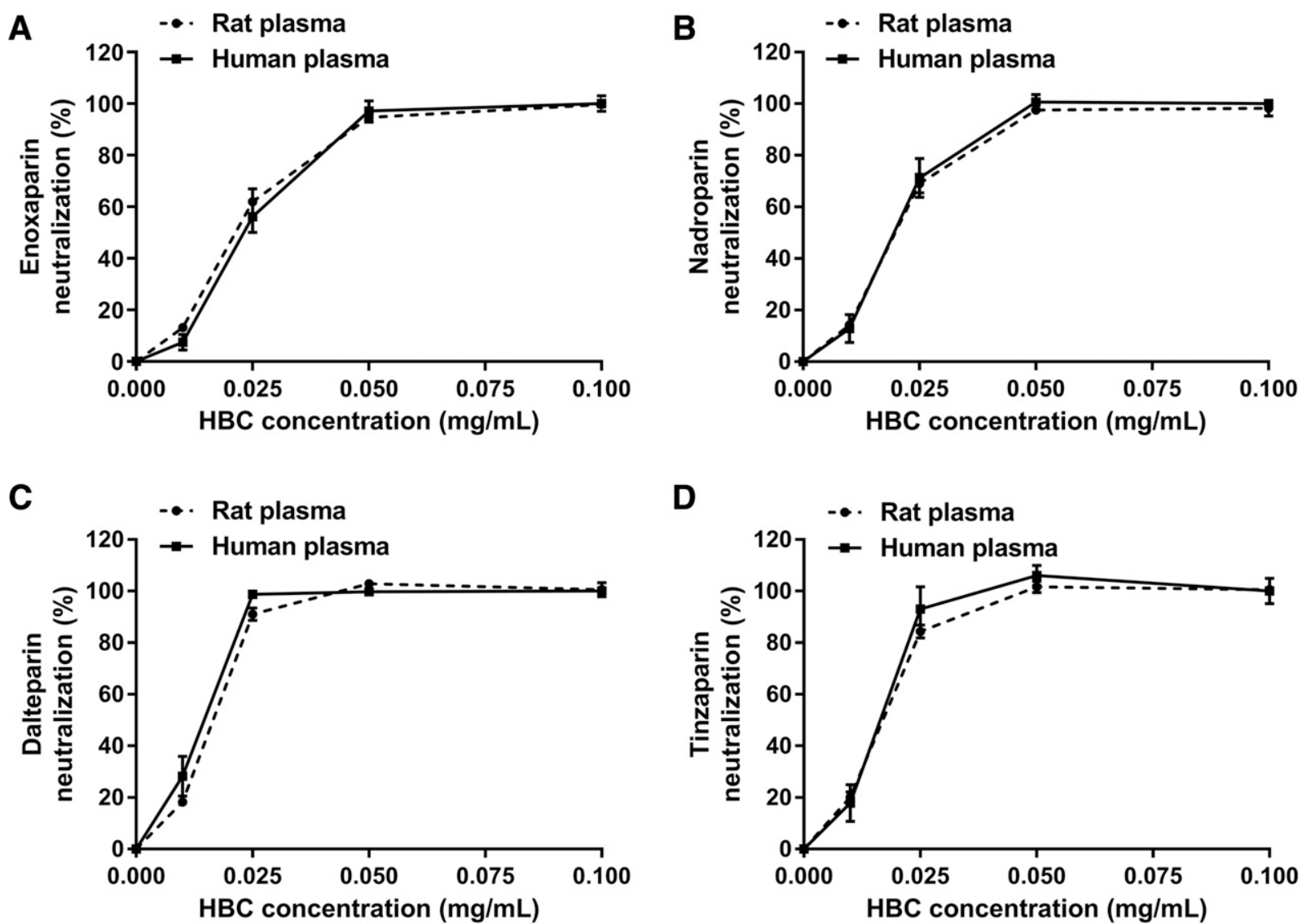

Fig. 3. Neutralization of enoxaparin (A), nadroparin (B), dalteparin (C), and tinzaparin (D) by HBC in sodium citrate-anticoagulated plasma with the antifactor Xa assay. Results are shown as mean \pm S.D.

Efficacy in Animals. All currently in vivo-studied LMWHs (tinzaparin, dalteparin, and nadroparin) prolonged bleeding time and increased antifactor Xa activity at all studied time points. HBC completely reversed the effects of LMWHs on bleeding time and antifactor Xa activity to the approximate control values in a 20 -minute window (Fig. 7). Our previous study demonstrated that HBC also reversed an increased bleeding time and antifactor Xa activity by enoxaparin in the same animal model (Kalaska et al., 2016). We observed an almost-complete reversal of bleeding time, and antifactor Xa activity increased by nadroparin 1 hour from the administration, whereas more than $60 \%$ of reversal activity was maintained after 2 hours (Fig. 8).

\section{Discussion}

We have previously shown the ability of HBC to neutralize UFH, enoxaparin, and fondaparinux. The complete reversal of enoxaparin demonstrated the potential advantage of $\mathrm{HBC}$ over protamine sulfate (Kalaska et al., 2016). In this work, we evaluate the safety profile of $\mathrm{HBC}$ and its reversal activity against dalteparin, nadroparin, and tinzaparin. We found that HBC bound all tested LMWHs forming the smallest complexes with dalteparin and tinzaparin. The efficacy and safety studies in rats showed that $\mathrm{HBC}$ could be a universal antidote for all LMWHs. We also confirmed the neutralization of all LMWHs by HBC in human plasma.

Our in vitro studies investigated the cardiomyocyte viability after exposure to HBC and PMAPTAC198 as a control to check the cytotoxicity reduction of the block copolymer compared with homopolymer. Reversal of heparins by cationic protamine sulfate can cause right ventricular failure and bradycardia (Ocal et al., 2005; Sokolowska et al., 2016) and may induce left ventricular dysfunction because of its direct effect on cardiomyocyte contractile processes (Hird et al., 1995). Because of the negative mitochondrial membrane potential in cardiomyocytes, cationic compounds may be taken up by mitochondria and may, therefore, lead to cardiomyocyte

TABLE 1

Zeta potential of LMWH-HBC complexes and thermodynamic parameters for the interaction of LMWHs with HBC

\begin{tabular}{|c|c|c|c|c|c|c|}
\hline & $\begin{array}{l}\text { Zeta Potential } \\
\quad(\mathrm{mV})\end{array}$ & $\begin{array}{l}\text { Stoichiometry/ } \\
\text { Number of } \\
\text { Binding Sites, } \boldsymbol{n}\end{array}$ & $\begin{array}{c}\text { Estimated Binding } \\
\text { Constant } \\
K_{a} \times 10^{-7}\left[\mathrm{M}^{-1}\right]\end{array}$ & $\begin{array}{c}\text { Estimated Heat } \\
\text { of Binding, } \boldsymbol{\Delta H} \\
{[\mathrm{kcal} / \mathrm{mol}]}\end{array}$ & $\begin{array}{c}\text { Gibbs Free } \\
\text { Energy, } \boldsymbol{\Delta} \boldsymbol{G} \\
{[\mathrm{kcal} / \mathrm{mol}]}\end{array}$ & $\begin{array}{c}\text { Change in } \\
\text { Entropy - T } \boldsymbol{\Delta} \boldsymbol{S}, \\
{[\mathrm{kcal} / \mathrm{mol}]}\end{array}$ \\
\hline Enoxaparin-HBC & $1.49 \pm 0.63$ & $0.47 \pm 0.02$ & $92.4 \pm 19.9$ & $8.35 \pm 0.54$ & -6.87 & -15.2 \\
\hline Nadroparin-HBC & $-3.93 \pm 0.29$ & $0.49 \pm 0.01$ & $10.8 \pm 2.14$ & $8.01 \pm 0.22$ & -8.14 & -16.2 \\
\hline Dalteparin-HBC & $-0.13 \pm 0.08$ & $0.38 \pm 0.01$ & $1.42 \pm 0.64$ & $9.86 \pm 0.22$ & -9.35 & -19.2 \\
\hline Tinzaparin-HBC & $-5.29 \pm 1.29$ & $0.69 \pm 0.01$ & $6.51 \pm 1.68$ & $8.68 \pm 0.26$ & -8.45 & -17.1 \\
\hline
\end{tabular}



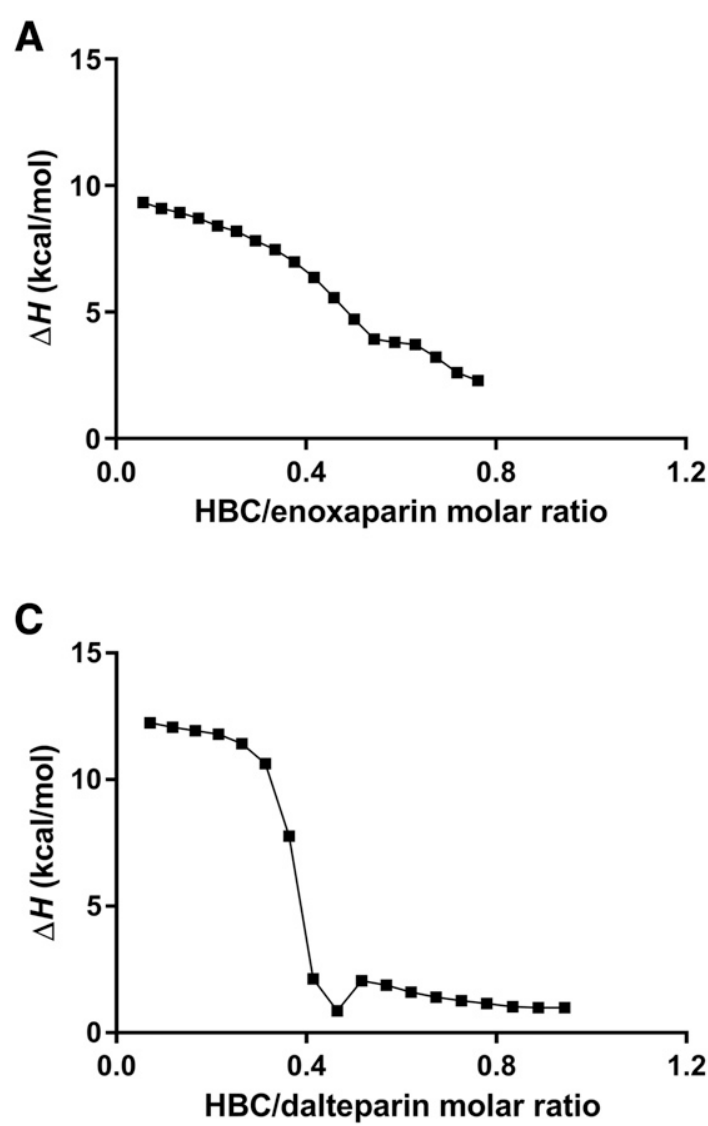
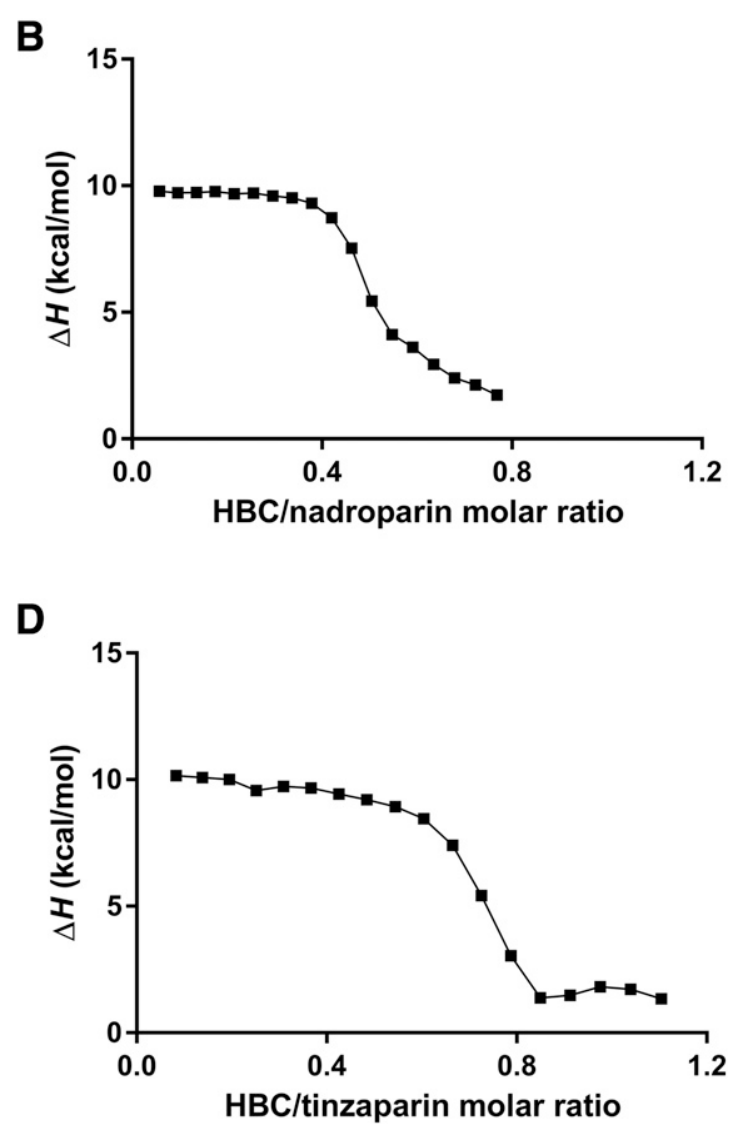

Fig. 4. Estimated heat of binding for the titration of HBC into enoxaparin (A), nadroparin (B), dalteparin (C), and tinzaparin (D).

damage (Hasinoff et al., 2003, 2016). Indeed, cationic PMAPTAC198 homopolymer decreased cardiomyocyte viability, whereas HBC had no significant effect on cell viability, confirming a well-known strategy of copolymerization or functionalization with PEG to reduce polycation toxicity (Pasut and Veronese, 2012). Though HBC and PMAPTAC198 did not affect human endothelial cells viability in vitro at concentrations up to $0.05 \mathrm{mg} / \mathrm{ml}$, endothelial cells seem to be more sensitive than cardiomyocytes to the effects of both polycationic compounds applied in the higher concentration. The reduced viability may result from their interaction with the anionic surface of endothelial cells, as was shown in the case of protamine sulfate (Chang and Voelkel, 1989).

$\mathrm{HBC}$ sufficiently reversed antifactor Xa activity increased in vitro by studied LMWHs in the concentration of $0.025 \mathrm{mg} / \mathrm{ml}$. In contrast, protamine sulfate was partially effective and showed an anticoagulant effect at concentrations higher than $0.05 \mathrm{mg} / \mathrm{ml}$ (Shenoi et al., 2014). The efficacious concentrations of HBC were three to six times lower than those maximums studied in the cardiomyocyte viability experiment. UHRAs demonstrated complete tinzaparin neutralization at similar ratios (Shenoi et al., 2014). Andexanet alfa in the concentration of $\sim 3 \mu \mathrm{M}$ also completely reversed enoxaparin (Lu et al., 2013). However, the authors focused on one LMWH (tinzaparin or enoxaparin) and have not investigated the cardiotoxicity.

We applied DLS and ITC techniques to understand the mechanism of interaction between HBC and LMWHs. The enthalpy of binding was positive, indicating an endothermic interaction. It was a bit unexpected for electrostatic interaction. However, endothermic interactions between oppositely charged polyelectrolytes have been reported (Ma et al., 2009). On the other hand, entropy values were positive, indicating that the neutralization process is entropy driven. The values of $n$ vary from 0.38 to 0.69 , indicating that one chain of HBC may bind more than one molecule of an LMWH (from 1.4 to 2.6 on the average). This is not surprising, taking into account that one HBC chain bears on the average 53 cationic groups, whereas the chains of the LMWHs bear from about 22 to 32 negative sulfate groups (assuming they all have the same charge density equal to 2.7 sulfate groups per disaccharide repeat unit, i.e., a charge of $-5 / \mathrm{kDa}$ ) (Kalathottukaren et al., 2017a). It means that the number of $\mathrm{HBC}$ cationic groups per chain is about 1.6-2.5 times greater than that of LMWHs anionic groups, which is close to $1 / n$, that is, the number of LMWH chains complexed by one HBC chain. Moreover, the value of $n$ for HBC-enoxaparin can be compared with the respective value for dendrimeric UHRA, for which the $n$ value varies from 0.17 to 0.28 (Shenoi et al., 2014), and indicates that one UHRA dendrimer may bind from 3.6 to 5.9 chains of enoxaparin. It is about two times more than the number of enoxaparin chains complexed by one HBC molecule. Considering that the molecular weight of UHRA is $>23 \mathrm{kDa}$ (i.e., almost twice that of $\mathrm{HBC}$ ), the ability to bind enoxaparin of UHRA and HBC is comparable in terms of enoxaparin chains bound per kiloDalton of a polycation. The binding constants for HBC-LMWH complexes are of the order of $10^{7}-10^{8}$, so the binding is rather strong also when compared 
A
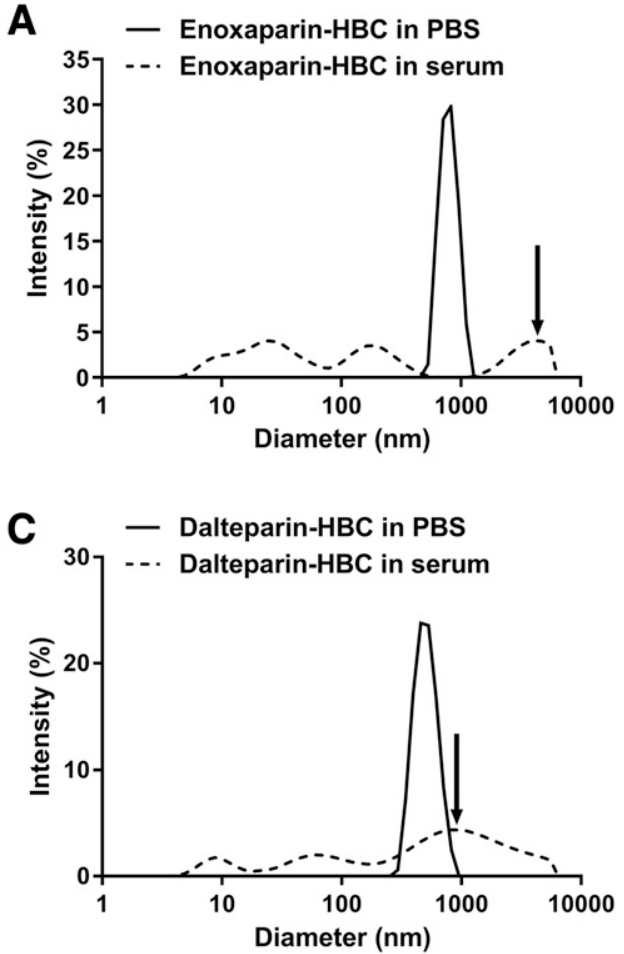

B

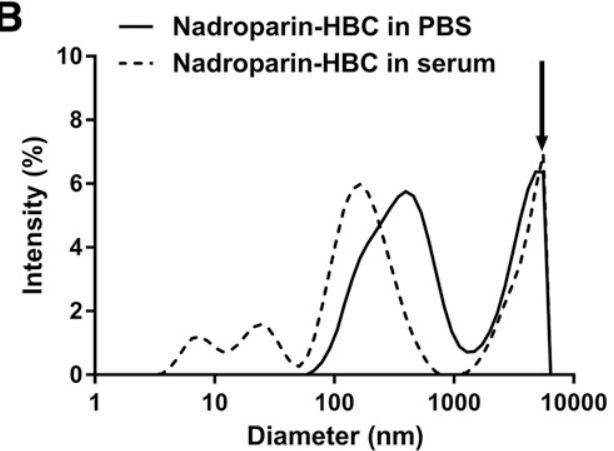

D

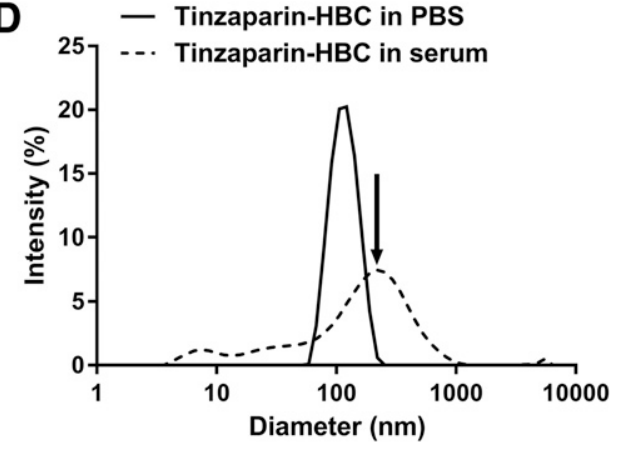

Fig. 5. The size profile of enoxaparin-HBC (A), nadroparin-HBC (B), dalteparin-HBC (C), and tinzaparin-HBC (D) complexes in the aqueous solutions (PBS, $\mathrm{pH}$ 7.4) and bovine serum, and PBS and HBC (E) in bovine serum obtained by the dynamic light scattering analysis.

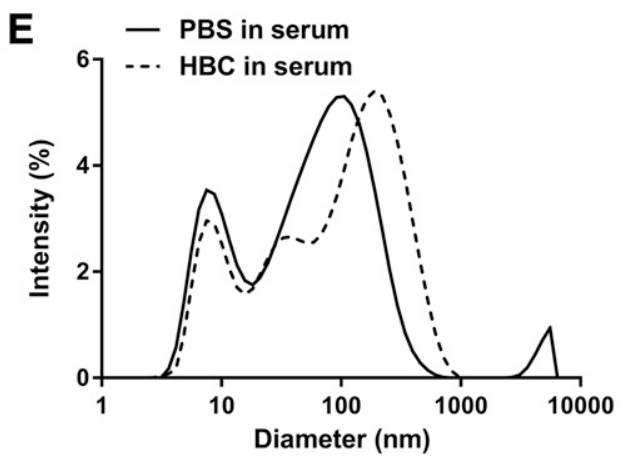

with UHRA-enoxaparin binding for which $K_{a}$ was of the order of $10^{4}-10^{5}$ (Shenoi et al., 2014).

The development of a reversal agent for LMWHs is a multistep process involving an evaluation of its safety. The nonclinical safety evaluation mainly includes a characterization of toxic effects, gross necropsy observations, dose dependence, and relationship to exposure. According to International Conference on Harmonisation guidelines, it

TABLE 2

Selected clinical and gross necropsy observations during safety study in rats

The values indicate a total number of observations/number of animals concerned.

\begin{tabular}{|c|c|c|c|c|c|}
\hline Dose $(\mathrm{mg} / \mathrm{kg})$ & 0 & 5 & 10 & 20 & 40 \\
\hline \multicolumn{6}{|l|}{ Selected clinical findings } \\
\hline Decreased locomotive activity & $0 / 7$ & $0 / 6$ & $0 / 6$ & $3 / 6$ & $10 / 10$ \\
\hline Apathy & $0 / 7$ & $0 / 6$ & $0 / 6$ & $3 / 6$ & $10 / 10$ \\
\hline Increased thirst & $0 / 7$ & $0 / 6$ & $0 / 6$ & $0 / 6$ & $4 / 10$ \\
\hline Secretion of porphyrins & $0 / 7$ & $1 / 6$ & $1 / 6$ & $0 / 6$ & $5 / 10$ \\
\hline Mild allergic reactions & $0 / 7$ & $1 / 6$ & $0 / 6$ & $5 / 6$ & $4 / 10$ \\
\hline Mortality & $0 / 7$ & $0 / 6$ & $0 / 6$ & $0 / 6$ & $0 / 10$ \\
\hline \multicolumn{6}{|c|}{ Selected gross necropsy findings } \\
\hline Lung congestion & $0 / 7$ & $1 / 6$ & $0 / 6$ & $1 / 6$ & $1 / 10$ \\
\hline Kidney congestion & $0 / 7$ & $0 / 6$ & $0 / 6$ & $0 / 6$ & $4 / 10$ \\
\hline Kidney color heterogeneity & $0 / 7$ & $1 / 6$ & $0 / 6$ & $0 / 6$ & $4 / 10$ \\
\hline Thymus congestion & $0 / 7$ & $0 / 6$ & $0 / 6$ & $1 / 6$ & $1 / 10$ \\
\hline Duodenum congestion & $0 / 7$ & $0 / 6$ & $0 / 6$ & $1 / 6$ & $0 / 10$ \\
\hline Pancreas congestion & $0 / 7$ & $0 / 6$ & $0 / 6$ & $1 / 6$ & $0 / 10$ \\
\hline Liver abnormalities & $0 / 7$ & $0 / 6$ & $0 / 6$ & $1 / 6$ & $1 / 10$ \\
\hline Urine sediments & $0 / 7$ & $0 / 6$ & $0 / 6$ & $0 / 6$ & $3 / 10$ \\
\hline
\end{tabular}


TABLE 3

Biochemical parameters in rats treated with $\mathrm{HBC}$ at the dose of $40 \mathrm{mg} / \mathrm{kg}$

Results are shown as a median with lower and upper limits.

\begin{tabular}{lcc}
\hline & Vehicle & HBC \\
\hline Alanine transaminase, U/l & $34.5(25.0-40.0)$ & $26.0(19.0-34.0)$ \\
Aspartate aminotransferase, U/l & $86.5(76.0-94.0)$ & $105.0(67.0-147.0)$ \\
Total bilirubin, mg/dl & $<0.15$ & $<0.15$ \\
Urea, mg/dl & $42.0(39.0-44.0)$ & $229.0(38.0-448.0)$ \\
Creatinine, mg/dl & $0.20(0.20-0.30)$ & $0.95(0.20-3.40)$ \\
\hline
\end{tabular}

should support first-in-human clinical trials . Nonclinical toxicity studies of the most promising LMWH-reversal agents, ciraparantag and andexanet alfa, have been conducted in different animal models. Ciraparantag was evaluated to perform the core safety pharmacology studies in rat and dog models. Based on the lack of adverse systemic toxicity and mortality, doses of ciraparantag up to $35 \mathrm{mg} / \mathrm{kg}$ in dogs and $40 \mathrm{mg} / \mathrm{kg}$ in rats were chosen as maximum tolerated doses. Ciraparantag in the single dose of $40 \mathrm{mg} / \mathrm{kg}$ was well tolerated in dogs. There were mild signs of hypersalivation and loss of balance. In rats, ciraparantag in a single dose of $40 \mathrm{mg} / \mathrm{kg}$ caused decreased activity and red-stained nares (Sullivan et al., 2015). Similar treatment-emergent adverse events were observed in healthy volunteers later (Ansell et al., 2016). The authors have not investigated LMWH-reversal effects of ciraparantag in humans. Andexanet alfa was tested in a single and repeat-dose toxicity study. It was administered to rats or monkeys alone and in combination with apixaban, rivaroxaban, or enoxaparin. There were no systemic or tissue pathologies in studied animals after single intravenous injections of up to $60 \mathrm{mg} / \mathrm{kg}$. In registration data available at Food and Drug Administration, five mortalities of rats treated with andexanet alfa twice daily for 14 days were observed. In monkeys, they found neither significant safety concerns nor unexpected outcomes. All observations were transient or reversible and included transient hypoactivity, labored respiration, vomiting, and anaphylactic reaction (Lu et al., 2017). Similarly to other antidotes (Kalathottukaren et al., 2017b), we tested the toxicity of HBC administered without heparin. Besides mild and occasional clinical and gross necropsy findings, single doses up to $20 \mathrm{mg} / \mathrm{kg}$ of $\mathrm{HBC}$ were well tolerated. We observed changes in rats' behavior and signs of organ damage in the group of rats treated with a dose of $40 \mathrm{mg} / \mathrm{kg}$ of HBC. In some of the rats, we performed additional biochemistry and histopathological analysis, which revealed a sign of acute tubular necrosis and increased biochemical markers of kidney damage in two cases. Nephrotoxicity may result from the binding of cationic $\mathrm{HBC}$ with an anionic surface of renal epithelial cells, similarly to the postulated chemical interactions between crystals and epithelial cell surfaces in kidney stone formation (Lieske et al., 1997; Wesson and Ward, 2006). Similarly to protamine, the efficacious dose of HBC for LMWHs reversal is closely related to the dose of studied LMWH and is based on the estimated optimal mass ratios. In the previous study (Kalaska et al., 2016), we used $\mathrm{HBC}$ at the dose of $6 \mathrm{mg} / \mathrm{kg}$ that corresponded to $3 \mathrm{mg} / \mathrm{kg}$ of enoxaparin. If we assume $3 \mathrm{mg} / \mathrm{kg}$ as a therapeutic dose of enoxaparin in rats, the multiple to the no-observed-adverseeffect-level and adverse level for HBC would be $\sim 4$ and $\sim 8$, respectively. LMWHs were administered in supratherapeutic doses to induce clinically relevant major bleeding. Indeed, anticoagulation by LMWHs increased bleeding time and antifactor Xa activity. The strong anticoagulant effect was observed in $100 \%$ of the animals. HBC administered in dose corresponding to 2.5:1 mass ratio, but not exceeding safety dose of $20 \mathrm{mg} / \mathrm{kg}$, completely reversed the effects of LMWHs on bleeding time and antifactor $\mathrm{Xa}$ activity to the approximate control values in a 20 -minute window. A similar study has been conducted to determine the reversal effect of andexanet alfa on enoxaparin. However, administration of andexanet alfa only partially reversed antifactor Xa activity and decreased bleeding in $\sim 80 \%$ of the animals in a rat
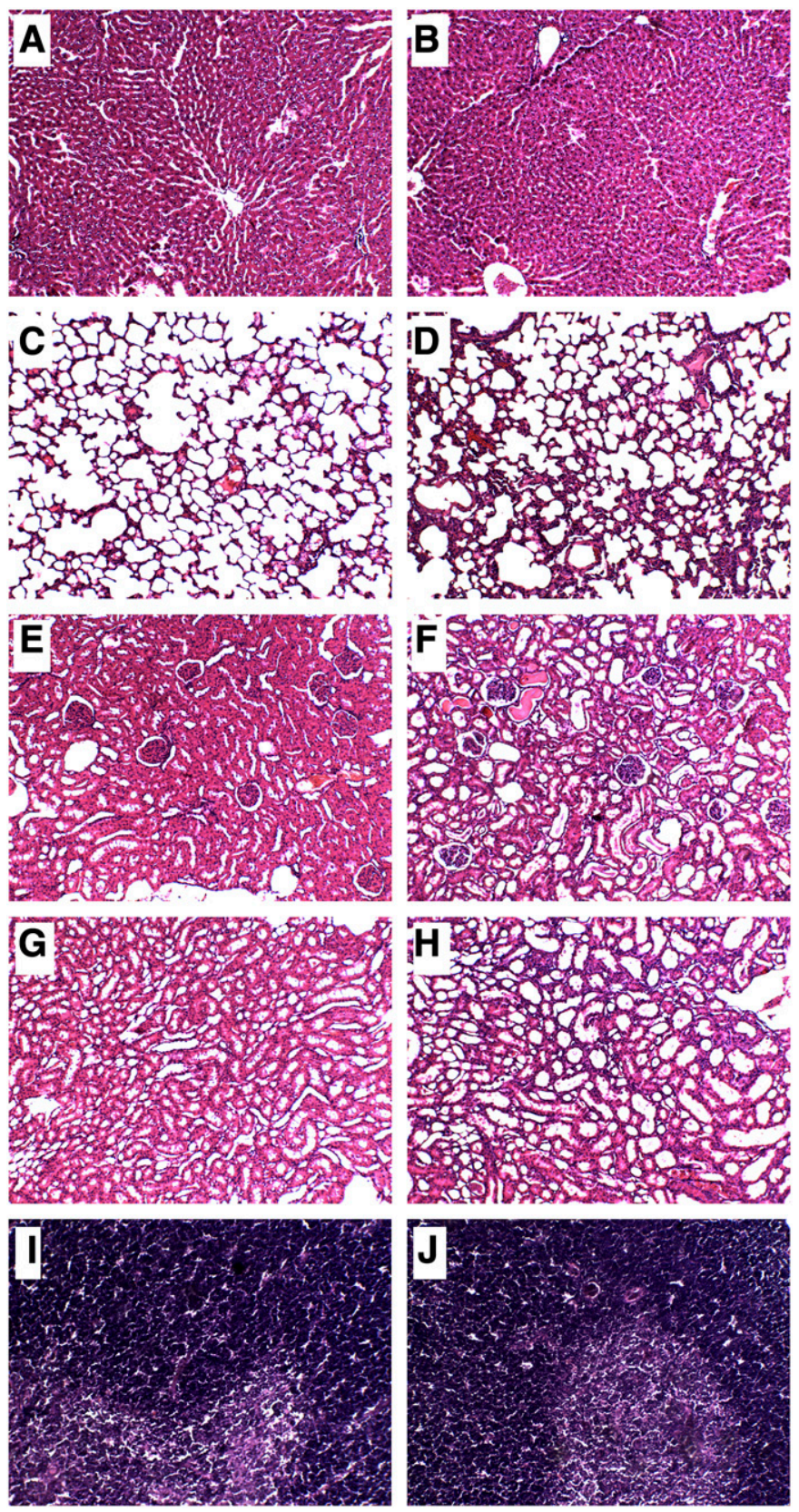

Fig. 6. Representative micrographs of tissue sections of liver (A and B), lung $(C$ and $D)$, renal cortex $(\mathrm{E}$ and $\mathrm{F})$, renal medulla $(\mathrm{G}$ and $\mathrm{H})$, and thymus (I and $\mathrm{J}$ ) 4 days after intravenous administration of PBS (left column; A, C, E, G, and I) and $\mathrm{HBC}$ at the dose of $40 \mathrm{mg} / \mathrm{kg}$ (right column; $\mathrm{B}, \mathrm{D}, \mathrm{F}, \mathrm{H}$, and J) in rats. Original magnification, $100 \times$; hematoxylin and eosin staining. 

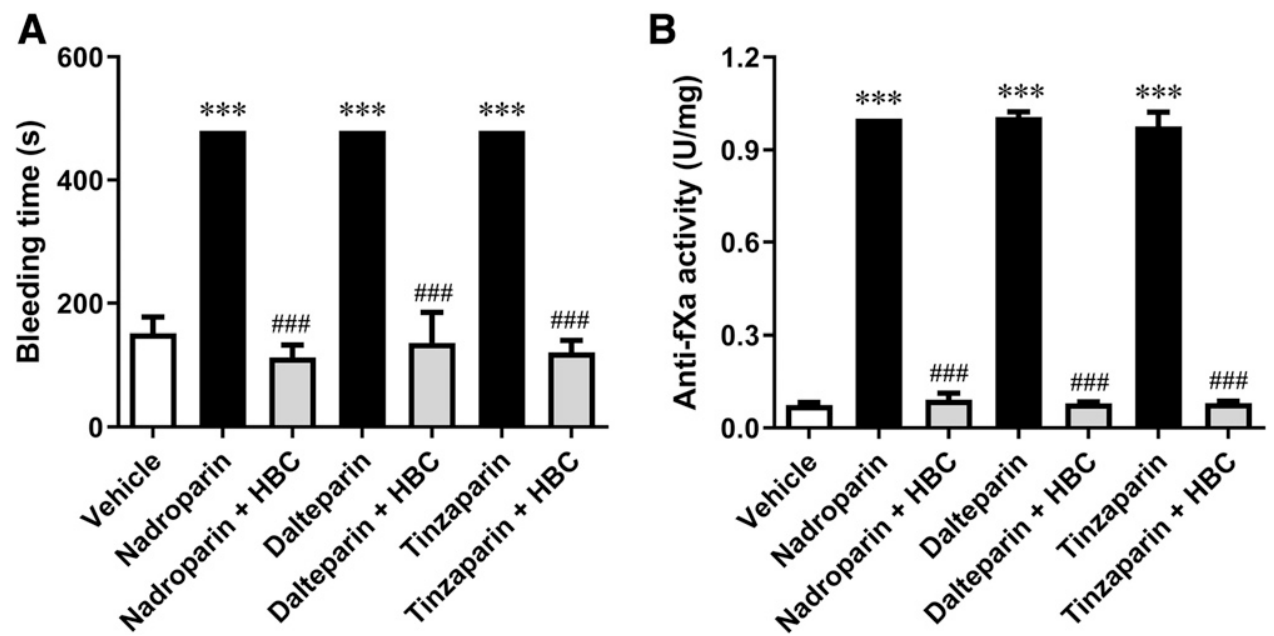

Fig. 7. Neutralization of nadroparin, dalteparin, and tinzaparin by $\mathrm{HBC}$ in rats as measured by bleeding time (A) and antifactor $\mathrm{Xa}$ activity (B). ${ }^{* * *} P<0.001$

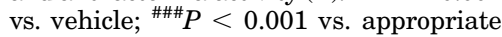
low-molecular-weight heparin, unpaired Student's $t$ test. Results are shown as mean \pm S.D. and analyzed with GraphPad Prism software 6

tail-transection model (Hollenbach et al., 2012). We further evaluated time-related changes of the reversal activity of HBC against nadroparin as an example, since HBC effect was comparable for all studied LMWHs. The almost-complete neutralization of bleeding time and antifactor Xa activity was observed for 1 hour after a 5-minute infusion of HBC, whereas more than $60 \%$ of its reversal activity was maintained after 2 hours. Based on the literature data (Ibrahim et al., 2017), the inhibition of factor Xa activity by LMWH expires after $\sim 6$ hours from intravenous administration into rats. Thus, if a reversal of anticoagulation longer than 2 hours is needed for surgical intervention or during uncontrolled bleeding, HBC should probably be administered second time or, similarly to andexanet alfa, should be kept infused till the end of surgery or bleeding. Andexanet alfa due to the short half-life ( $\sim 1$ hour) had to be infused additionally to nonbleeding healthy volunteers to reverse activity of enoxaparin (Crowther et al., 2014, Siegal et al., 2015). In the ANNEXA-4 trial, andexanet alfa was administered as an intravenous bolus followed by a 2-hour infusion to 352 patients who had major acute bleeding after administration of rivaroxaban, apixaban, or enoxaparin. Of the 15 patients treated with enoxaparin who could be evaluated for anticoagulation efficacy, $13(87 \%)$ were adjudicated as having at least good hemostatic efficacy 12 hours after andexanet alfa infusion (Connolly et al., 2019). Thus, a 2-hour interval seems to be a convenient therapeutic window for the reversing of LMWHs in patients, especially those with a high risk of thrombosis, in which restoring of anticoagulant protection is needed. In the case of LMWHs reversal, more clinical trials are required to assess the effects of andexanet alfa on this drug-related bleeding (Sartori and Cosmi, 2018). Ciraparantag, another candidate for LMWHs reversal, was evaluated in rats and healthy volunteers. It stopped tail bleeding in rats treated with enoxaparin, and the effect was stronger than that of protamine sulfate (Laulicht et al., 2013). In healthy volunteers, ciraparantag entirely and rapidly reversed the clotting time induced by enoxaparin but also caused adverse events in more than half of patients (Ansell et al., 2016).

Based on the previous studies on enoxaparin, one could assume that other LMWHs would also be neutralized by HBC. However, it would be impossible to conclude that $\mathrm{HBC}$ is an efficient and safe reversal agent for all main LMWHs without clear direct experimental evidence, especially because other
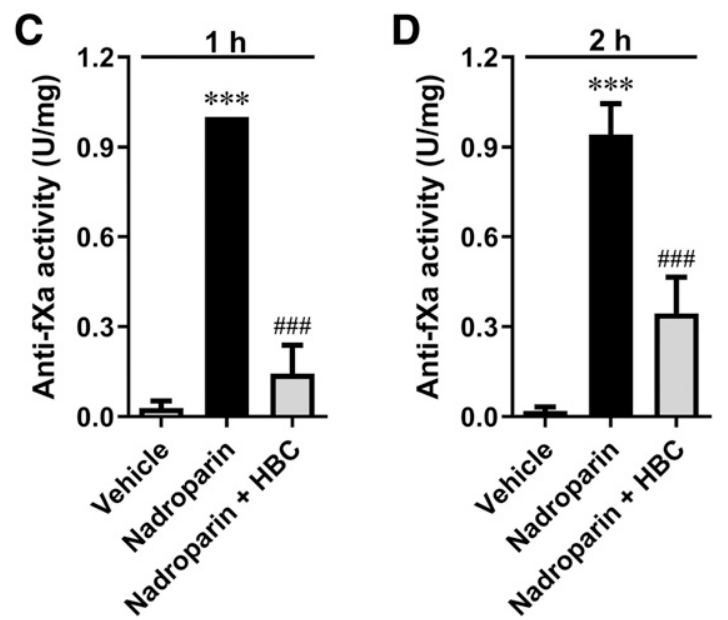

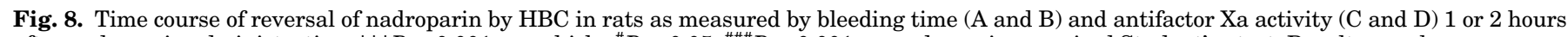

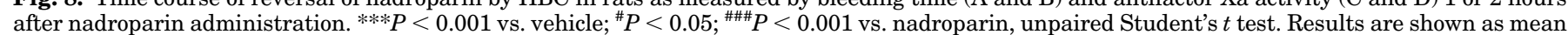
\pm S.D. and analyzed with GraphPad Prism software 6. 
antidotes were less effective against heparins with shorter chains. We also expanded the panel of safety studies in rodents and the toxicity in cardiomyocytes and characterized in more detail the mechanism of binding. We confirmed the efficacy of HBC against LMWHs in human plasma and its safety in HUVECs. It seems that the reversal activity of $\mathrm{HBC}$ after a single injection could last longer than the effect of andexanet alfa, which needs to be infused to maintain activity. Nevertheless, the second injection or prolonged infusion of HBC may be required in some clinical scenarios depending on how much time has passed since LMWHs injection and how much time is needed for surgical intervention. Thus, the evaluation of repeated-dose toxicity together with the estimation of the optimal route of $\mathrm{HBC}$ administration in nonhuman primates is required before the administration of $\mathrm{HBC}$ to patients.

To sum up, we present here a new pharmacological tool for binding all heparins. The studies with protamine, andexanet alfa, and ciraparantag show that there is still space for a more efficient, safer, and cost-effective reversal agent indicated for LMWHs, and yet no such antidote has been currently registered. The results of safety experiments point to a therapeutic potential of $\mathrm{HBC}$ administered in doses up to $20 \mathrm{mg} / \mathrm{kg}$. We estimated the neutralization weight ratio of HBC to LMWH for 2.5:1 in rats. Then, we confirmed it in human plasma. Importantly, HBC stopped bleeding from tail of heparinized rats and brought to control level typical coagulation parameters used in the clinical practice. HBC retained $\sim 80 \%$ and $\sim 60 \%$ of reversal activity after 1 and 2 hours, respectively. Based on this study, we may expect similar effect of HBC in patients treated with LMWHs.

\section{Acknowledgments}

We would like to thank Marcin Szczeciński, Piotr Rózga, and Marcin Feder from Adamed Pharma S.A. (Poland) for the critical review of the results and performing experiment in HUVECs.

\section{Authorship Contributions}

Participated in research design: Kalaska, Szczubiałka, Mogielnicki. Conducted experiments: Kalaska, Miklosz, Kamiński, Swieton, Jakimczuk, Yusa, Mogielnicki.

Contributed new reagents or analytic tools: Kalaska, Miklosz, Kamiński, Swieton, Yusa, Szczubiałka, Mogielnicki.

Performed data analysis: Kalaska, Kamiński, Swieton, Mogielnicki.

Wrote or contributed to the writing of the manuscript: Kalaska, Miklosz, Kamiński, Swieton, Jakimczuk, Yusa, Pawlak, Nowakowska, Szczubiałka, Mogielnicki.

\section{References}

Ansell JE, Laulicht BE, Bakhru SH, Hoffman M, Steiner SS, and Costin JC (2016) Ciraparantag safely and completely reverses the anticoagulant effects of low molecular weight heparin. Thromb Res 146:113-118.

Chang SW and Voelkel NF (1989) Charge-related lung microvascular injury. Am Rev Respir Dis 139:534-545.

Connolly SJ, Crowther M, Eikelboom JW, Gibson CM, Curnutte JT, Lawrence JH, Yue P, Bronson MD, Lu G, Conley PB, et al.; ANNEXA-4 Investigators (2019) Full study report of Andexanet Alfa for bleeding associated with factor Xa inhibitors. N Engl J Med 380:1326-1335.

Crowther M, Lu G, Conley P, Leeds J, Castillo J, Levy G, and Connolly S (2014) Reversal of factor Xa inhibitors-induced anticoagulation in healthy subjects by andexanet alfa. Crit Care Med 42:A1469.

Crowther MA and Warkentin TE (2008) Bleeding risk and the management of bleeding complications in patients undergoing anticoagulant therapy: focus on new anticoagulant agents. Blood 111:4871-4879.

Garcia DA, Baglin TP, Weitz JI, and Samama MM (2012) Parenteral anticoagulants: antithrombotic therapy and prevention of thrombosis, 9th ed: American college of chest physicians evidence-based clinical practice guidelines [published correction appears in Chest (2012) 141:1369; Chest (2013) 144:721]. Chest 141:e24S-e43S.

Gray E, Mulloy B, and Barrowcliffe TW (2008) Heparin and low-molecular-weight heparin. Thromb Haemost 99:807-818.
Hasinoff BB, Schnabl KL, Marusak RA, Patel D, and Huebner E (2003) Dexrazoxane (ICRF-187) protects cardiac myocytes against doxorubicin by preventing damage to mitochondria. Cardiovasc Toxicol 3:89-99.

Hasinoff BB, Wu X, Patel D, Kanagasabai R, Karmahapatra S, and Yalowich JC (2016) Mechanisms of action and reduced cardiotoxicity of pixantrone; a topoisomerase II targeting agent with cellular selectivity for the topoisomerase II $\alpha$ Isoform. J Pharmacol Exp Ther 356:397-409.

Heo YA (2018) Andexanet alfa: first global approval. Drugs 78:1049-1055.

Hird RB, Wakefield TW, Mukherjee R, Jones BU, Crawford FA, Andrews PC, Stanley JC, and Spinale FG (1995) Direct effects of protamine sulfate on myocyte contractile processes. Cellular and molecular mechanisms. Circulation 92 (9 Suppl): II433-II446.

Hollenbach SJ, Lu G, Deguzman F, Curnutte J, Conley PB, and Sinha U (2012) Bolus administration of PRT064445, a recombinant factor Xa inhibitor antidote, reverses blood loss and PD markers in a rat model following enoxaparin-induced anticoagulation. Eur Heart $J$ 33:309-310.

Hull RD, Pineo GF, Francis C, Bergqvist D, Fellenius C, Soderberg K, Holmqvist A, Mant M, Dear R, Baylis B, et al.; The North American Fragmin Trial Investigators (2000) Low-molecular-weight heparin prophylaxis using dalteparin in close proximity to surgery vs warfarin in hip arthroplasty patients: a double-blind, randomized comparison. Arch Intern Med 160:2199-2207.

Ibrahim SS, Osman R, Awad GAS, Mortada ND, and Geneidi AS (2017) Polysaccharides-based nanocomplexes for the prolonged delivery of enoxaparin: invitro and in-vivo evaluation. Int $J$ Pharm 526:271-279.

Kalaska B, Kamiński K, Miklosz J, Nakai K, Yusa SI, Pawlak D, Nowakowska M, Mogielnicki A, and Szczubiałka K (2018) Anticoagulant properties of poly(sodium 2-(acrylamido)-2-methylpropanesulfonate)-based di- and triblock polymers. Biomacromolecules 19:3104-3118.

Kalaska B, Kaminski K, Miklosz J, Yusa SI, Sokolowska E, Blazejczyk A, Wietrzyk J, Kasacka I, Szczubialka K, Pawlak D, et al. (2016) Heparin-binding copolymer reverses effects of unfractionated heparin, enoxaparin, and fondaparinux in rats and mice. Transl Res 177:98-112.e10.

Kalaska B, Miklosz J, Kaminski K, Musielak B, Yusa SI, Pawlak D, Nowakowska M, Szczubiałka K, and Mogielnicki A (2019) The neutralization of heparan sulfate by heparin-binding copolymer as a potential therapeutic target. RSC Adv 9: $3020-3029$

Kalaska B, Kaminski K, Sokolowska E, Czaplicki D, Kujdowicz M, Stalinska K, Bereta J, Szczubialka K, Pawlak D, Nowakowska M, et al. (2015) Nonclinical evaluation of novel cationically modified polysaccharide antidotes for unfractionated heparin. PLoS ONE 10 (3):e0119486, doi: 10.1371/journal.pone.0119486 25781030.

Kalathottukaren MT, Abbina S, Yu K, Shenoi RA, Creagh AL, Haynes C, and Kizhakkedathu JN (2017a) A polymer therapeutic having universal heparin reversal activity: molecular design and functional mechanism. Biomacromolecules 18:3343-3358. Kalathottukaren MT, Abraham L, Kapopara PR, Lai BF, Shenoi RA, Rosell FI, Conway EM, Pryzdial EL, Morrissey JH, Haynes CA, et al. (2017b) Alteration of blood clotting and lung damage by protamine are avoided using the heparin and polyphosphate inhibitor UHRA. Blood 129:1368-1379.

Kalathottukaren MT, Creagh AL, Abbina S, Lu G, Karbarz MJ, Pandey A, Conley PB, Kizhakkedathu JN, and Haynes C (2018) Comparison of reversal activity and mechanism of action of UHRA, andexanet, and PER977 on heparin and oral FXa inhibitors. Blood Adv 2:2104-2114.

Kearon C, Akl EA, Comerota AJ, Prandoni P, Bounameaux H, Goldhaber SZ, Nelson ME, Wells PS, Gould MK, Dentali F, et al. (2012) Antithrombotic therapy for VTE disease: Antithrombotic Therapy and Prevention of Thrombosis, 9th ed: American College of Chest Physicians Evidence-Based Clinical Practice Guidelines, Chest 141:e419S-e496S.

Laulicht B, Bakhru S, Jiang X, Chen L, Pan D, Grosso M, Morishima Y, Brown K, Masumoto H, Costin J, et al. (2013) Antidote for new oral anticoagulants: mechanism of action and binding specificity of PER977. J Thromb Haemost 11:75.

Lieske JC, Norris R, and Toback FG (1997) Adhesion of hydroxyapatite crystals to anionic sites on the surface of renal epithelial cells. Am J Physiol 273:F224-F233.

Lu G, DeGuzman FR, Hollenbach SJ, Karbarz MJ, Abe K, Lee G, Luan P, Hutchaleelaha A, Inagaki M, Conley PB, et al. (2013) A specific antidote for reversal of anticoagulation by direct and indirect inhibitors of coagulation factor Xa. Nat Med 19:446-451.

Lu G, Hollenbach SJ, Baker DC, Tan S, Hutchaleelaha A, Curnutte JT, and Conley PB (2017) Preclinical safety and efficacy of andexanet alfa in animal models. $J$ Thromb Haemost 15:1747-1756.

Ma PL, Lavertu M, Winnik FM, and Buschmann MD (2009) New insights into chitosan-DNA interactions using isothermal titration microcalorimetry. Biomacromolecules 10:1490-1499.

Mismetti P, Quenet S, Levine M, Merli G, Decousus H, Derobert E, and Laporte S (2005) Enoxaparin in the treatment of deep vein thrombosis with or without pulmonary embolism: an individual patient data meta-analysis. Chest 128:2203-2210.

Ocal A, Kiriş I, Erdinç M, Peker O, Yavuz T, and Ibrişim E (2005) Efficiency of prostacyclin in the treatment of protamine-mediated right ventricular failure and acute pulmonary hypertension. Tohoku J Exp Med 207:51-58.

Pasut G and Veronese FM (2012) State of the art in PEGylation: the great versatility achieved after forty years of research. J Control Release 161:461-472.

Petersen JL, Mahaffey KW, Hasselblad V, Antman EM, Cohen M, Goodman SG, Langer A, Blazing MA, Le-Moigne-Amrani A, de Lemos JA, et al. (2004) Efficacy and bleeding complications among patients randomized to enoxaparin or unfractionated heparin for antithrombin therapy in non-ST-Segment elevation acute coronary syndromes: a systematic overview. JAMA 292:89-96.

Popiołek I, Niziołek A, Kamiński K, Kwolek U, Nowakowska M, and Szczubiałka K (2019) Cellular delivery and enhanced anticancer activity of berberine complexed with a cationic derivative of $\gamma$-cyclodextrin. Bioorg Med Chem 27:1414-1420.

Sartori M and Cosmi B (2018) Andexanet alfa to reverse the anticoagulant activity of factor Xa inhibitors: a review of design, development and potential place in therapy. J Thromb Thrombolysis 45:345-352. 
Shenoi RA, Kalathottukaren MT, Travers RJ, Lai BF, Creagh AL, Lange D, Yu K, Weinhart M, Chew BH, Du C, et al. (2014) Affinity-based design of a synthetic universal reversal agent for heparin anticoagulants. Sci Transl Med 6:260ra150.

Siegal DM, Curnutte JT, Connolly SJ, Lu G, Conley PB, Wiens BL, Mathur VS, Castillo J, Bronson MD, Leeds JM, et al. (2015) Andexanet alfa for the reversal of factor Xa inhibitor activity. $N$ Engl J Med 373:2413-2424

Simonneau G, Sors H, Charbonnier B, Page Y, Laaban JP, Azarian R, Laurent M, Hirsch JL, Ferrari E, Bosson JL, et al. (1997) A comparison of low-molecular-weight heparin with unfractionated heparin for acute pulmonary embolism. The THESEE Study Group. Tinzaparine ou Heparine Standard: Evaluations dans l'Embolie Pulmonaire. N Engl J Med 337:663-669.

Sokolowska E, Kalaska B, Miklosz J, and Mogielnicki A (2016) The toxicology of heparin reversal with protamine: past, present and future. Expert Opin Drug Metab Toxicol 12:897-909.

Sullivan DW Jr, Gad SC, Laulicht B, Bakhru S, and Steiner S (2015) Nonclinical safety assessment of PER977: a small molecule reversal agent for new oral anticoagulants and heparins. Int $J$ Toxicol 34:308-317.

Turpie AG, Bauer KA, Eriksson BI, and Lassen MR (2002) Fondaparinux vs enoxaparin for the prevention of venous thromboembolism in major orthopedic surgery: a meta-analysis of 4 randomized double-blind studies. Arch Intern Med 162 1833-1840.

van Rein N, Biedermann JS, van der Meer FJM, Cannegieter SC, Wiersma N, Vermaas HW, Reitsma PH, Kruip MJHA, and Lijfering WM (2017) Major bleeding risks of different low-molecular-weight heparin agents: a cohort study in 12934 patients treated for acute venous thrombosis. J Thromb Haemost 15:1386-1391.

van Veen JJ, Maclean RM, Hampton KK, Laidlaw S, Kitchen S, Toth P, and Makris M (2011) Protamine reversal of low molecular weight heparin: clinically effective? Blood Coagul Fibrinolysis 22:565-570.

Wesson JA and Ward MD (2006) Role of crystal surface adhesion in kidney stone disease. Curr Opin Nephrol Hypertens 15:386-393.

Address correspondence to: Bartlomiej Kalaska, Department of Pharmacodynamics, Medical University of Bialystok, Mickiewicza 2c, 15-089, Bialystok, Poland. E-mail: bartlomiej.kalaska@umb.edu.pl; or Krzysztof Szczubiałka, Faculty of Chemistry, Jagiellonian University, Gronostajowa 2, 30-387 Krakow, Poland. E-mail: szczubia@chemia.uj.edu.pl 